Classification

Physics Abstracts

$07.80-61.16 \mathrm{D}-06.50$

\title{
Factors affecting the accuracy of elemental analysis by transmission EELS
}

\author{
Raymond F. Egerton $\left(^{*}\right)$ \\ Department of Materials Science and Engineering, State University of New York, Stony Brook, NY \\ 11794, U.S.A.
}

(Received October 24, 1990; accepted January 28, 1991)

\begin{abstract}
The accuracy of elemental analysis by electron energy-loss spectroscopy depends on many factors, such as the concentration of the elements being analysed and the energies of the corresponding ionization edges. We discuss sources of inaccuracy in the acquisition and analysis of spectra, in the quantification procedure and in the required ionization cross sections, together with procedures for minimising these errors.
\end{abstract}

\section{Introduction.}

In electron energy-loss spectroscopy (EELS), elemental analysis is achieved by recording the number of electrons which have suffered an energy loss E during transmission through a thin specimen, particularly via the process of inner-shell ionization. The amount of each element (within the irradiated volume of specimen) is proportional to its characteristic-loss signal, usually measured as an area $I_{c}(\beta, \Delta)$ integrated over an energy range $\Delta$ beyond the corresponding ionization edge; see figure 1a.

Absolute quantification of the areal density $N$ of a particular element (the number of atoms per unit area of specimen) utilises the approximate formula [1]:

$$
I_{\mathrm{c}}(\beta, \Delta) \approx N \sigma_{\mathrm{c}}(\beta, \Delta) I_{1}(\beta, \Delta)
$$

where $\sigma_{\mathbf{c}}(\beta, \Delta)$ is a cross section for inner-shell scattering, appropriate to the range of integration $\Delta$ and the angular range $\beta$ of scattering collected by the electron spectrometer (defined by an angle-limiting aperture in the TEM column); $I_{1}(\beta, \Delta)$ is the low-loss intensity, integrated up to $E=\Delta$. For most purposes, only elemental ratios are required; if so, $I_{1}$ is the same for each element and need not be measured.

The accuracy of elemental analysis can be discussed in terms of the various symbols appearing in equation (1).

$\left({ }^{*}\right)$ Address from July 1991: Physics Department, University of Alberta, Edmonton, Canada T6G 2J1. 
(a)

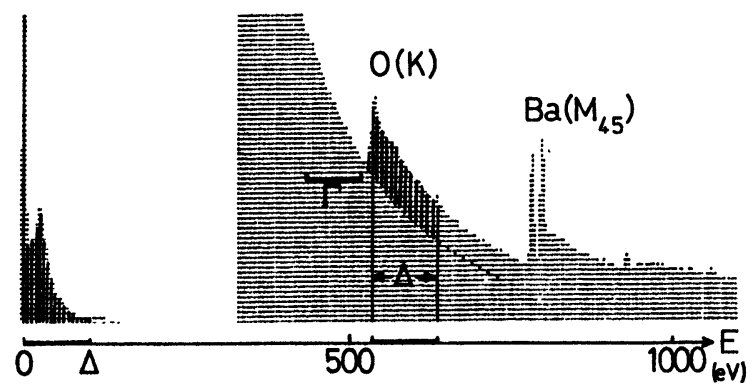

(b)

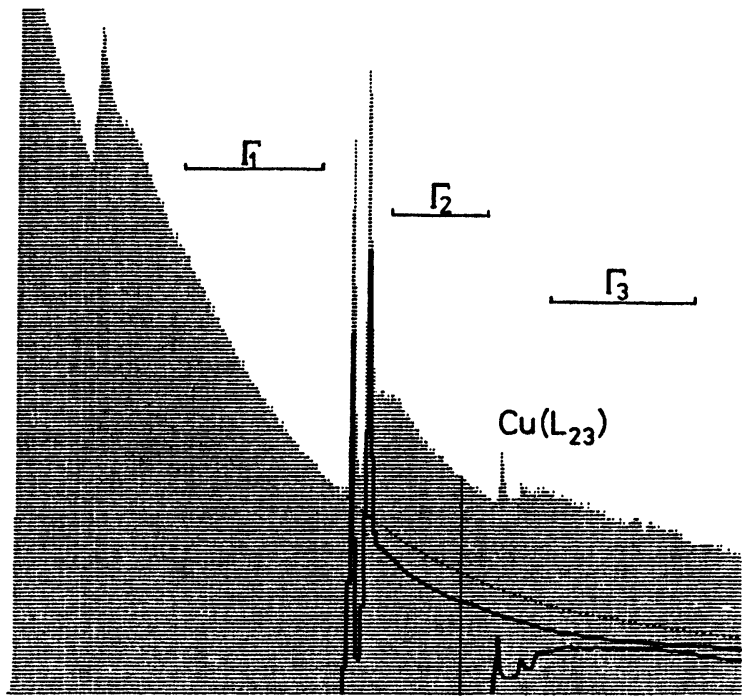

Fig. 1. - Energy-loss spectrum of YBCO superconductor, illustrating two methods of measuring elemental concentration: (a) the conventional method of background fitting, extrapolation and subtraction; (b) singlestage (background + edge) fitting over regions below and above each threshold energy. Fitting regions are denoted $\Gamma$.

Measurement errors derive mainly from the difficulty in measuring the relatively weak core-loss intensity $I_{\mathbf{c}}$ (the low-loss signal $I_{1}$ presents less of a problem).

Procedural errors result from the approximations involved in equation (1), due to the influence of other scattering processes (elastic and low-energy inelastic scattering).

Errors in cross section reflect uncertainties in the values of $\sigma_{\mathfrak{c}}$, arising from assumptions made in its calculation or experimental errors in its measurement.

\section{Measurement errors.}

The problems in accurately measuring core-loss intensity arise from the fact that the signal at higher energy loss is relatively weak. Shot noise provides a fundamental limit to the accuracy obtainable; its effect is augmented by the fact that, in the standard analysis procedure, the background preceding the edge must be extrapolated and subtracted from the spectral intensity in order to derive $I_{\mathbf{c}}$. Systematic and statistical errors of extrapolation [2] are particularly severe in the case of weak edges on a large background, or where two edges are closely spaced in energy. 
2.1 BACKGROUND MODELLING. - The form of the background contribution underlying an ionization edge is usually obtained by fitting the intensity immediately preceding the edge to a simple function of energy loss $E$, with adjustable parameters. The form $A E^{-r}$ is popular because it contains only two parameters ( $A$ and $r$ ), has a physical basis in terms of scattering theory and yields a reasonable fit in many cases.

Linear least-squares fitting (on logarithmic coordinates) is widely used because simple algorithms are available [3]. Other fitting procedures, such as a weighted least-squares fit $[4,5]$, ravinesearch and simplex procedures $[6,7]$ have been implemented where a very high background component demands increased accuracy.

Alternative background-modelling functions (e.g. exponential, polynomial) have sometimes given better accuracy [8]. In the case of ionization edges below about $100 \mathrm{eV}$ loss, the $A E^{-r}$ model can be expected to fail, even if the data has been deconvolved to remove plural scattering; fitting the tail of the low-loss peak to a Drude-type expression provides a more realistic alternative [9].

2.2 MODELLING OF THE BACKGROUND + EDGE. - Background fitting is a severe problem if adjacent ionization edges are closely spaced. Then the fitting region is of restricted width, so that statistical errors of extrapolation are increased [2]. Furthermore, the background may not decrease smoothly in the fitting region, because of plural scattering or fine-structure modulations of the preceding edge, leading to systematic error in the extrapolation. A proposed solution [10] is to effectively extend the fitting region beyond the ionization threshold by matching the spectral intensity $J(E)$ to a weighted sum of background and core-loss contributions:

$$
J(E)=k\left(\mathrm{~d} \sigma_{\mathrm{c}} / \mathrm{d} E\right)+A E^{-r}
$$

Here, $\mathrm{d} \sigma_{\mathrm{c}} / \mathrm{d} E$ is the energy-differential cross section for core-loss scattering and $k$ represents the relative concentration of the element concerned. Values of $k, A$ and $r$ can be found by leastsquares fitting above and below the threshold energy; in general, one would expect a closer fit if the region just above the threshold (where the intensity is influenced by solid-state effects) is omitted from the fitting procedure; see figure $1 \mathrm{~b}$.

Absolute values of the $\mathrm{d} \sigma_{\mathrm{c}} / \mathrm{d} E$ can be calculated by hydrogenic or Hartree-Slater models; if $J(E)$ has been deconvolved to remove plural scattering, $k=N I_{0}$ in equation (2), where $I_{0}$ is the zero-loss intensity, leading to an absolute estimate of the areal density $N$. Alternatively, edge shapes can be measured from standards of known composition, convolved with the low-loss spectrum if necessary to allow for plural scattering [11]. Another possibility (Fig. 2) is to fit first - or second-differentials of the spectral data [12], as is done in x-ray emission spectroscopy.

One possible limit to accuracy and sensitivity of elemental detection is the presence of extended fine-structure modulations in preceding edges; see figure 2. To accurately account for these, the edge profiles may have to be recorded from atomic species which are in similar crystallographic environments to those in the analysed sample.

2.3 LENS ABERRATIONS. - If EELS is carried out in a conventional transmission electron microscope (CTEM), electrons transmitted through the specimen must pass through several electron lenses before reaching the spectrometer. Aberrations of these lenses can degrade the energy-loss spectrum and the accuracy of analysis.

The literature contains several discussions of the effect of lens aberrations in EELS but the reader can easily be misled. Early papers deal with the situation where the projector lens is switched off for energy-loss spectroscopy [16]. More commonly, this lens is left on and the previously-named diffraction mode of spectroscopy (now known as diffraction coupling) corresponds to 


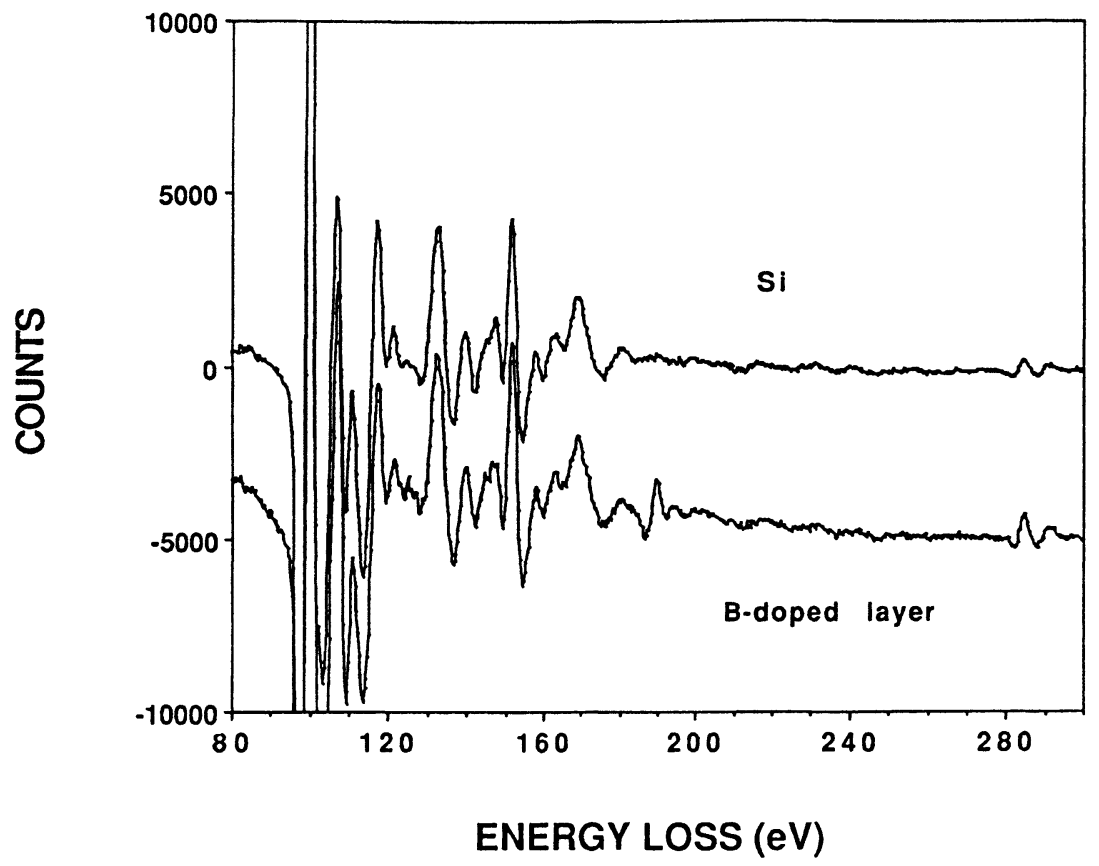

Fig. 2. - Second-difference spectra of pure Si and of 1\% B-doped Si, showing a boron K-edge at $188 \mathrm{eV}$, preceded by EXELFS oscillations of the silicon L-edge. Spectra recorded by R. Leapman using HB501 STEM and Gatan PEELS; specimen provided by R. Kola.

image mode of the microscope [17]. Spherical aberration of the objective lens can be significant, particularly in determining the spatial resolution of EELS and where the collection angle $\beta$ is large [17]. Chromatic aberration of post-specimen lenses is more important in determining energy resolution and the accuracy of elemental analysis.

Several publications $[16,17]$ treat the effect of lens aberrations at the spectrometer object plane. In modern CTEM systems, this plane (denoted SO in Fig. 3) is at the level of the projector-lens crossover, typically $40 \mathrm{~cm}$ above the CTEM screen. Because the spectrometer images this object at its exit plane SI (containing the energy-loss spectrum), chromatic broadening in the object results in a blurring of the spectrum. The loss in energy resolution may be serious for fine-structure studies, but has only a minor effect on accuracy of elemental analysis, provided the resolution remains better than $5 \mathrm{eV}$.

More important from the viewpoint of accuracy is the effect of aberrations at planes containing an aperture. In a CTEM, this could mean the objective, selected-area or spectrometer-entrance apertures. Of prime concern (since it occurs after at least three electron lenses) is the spectrometer entrance aperture (SEA in Fig. 3), located just below the microscope viewing screen. Irrespective of whether the screen is displaying a magnified image or a diffraction pattern, this aperture cuts off some electrons and therefore determines the amplitude of the energy loss signal and the collection efficiency. Chromatic aberration causes the central diffraction spot, or the image of a single point in the specimen, to be broadened into a disk of confusion, reducing the collection efficiency if the radius $R_{\mathrm{c}}$ of this disk exceeds the radius $R$ of the aperture; see figure 4a.

If a diffraction pattern is present on the CTEM screen (with camera length $L$ at the SEA plane):

$$
R_{\mathrm{c}}=\left(L d / f^{2}\right)\left(E / E_{0}\right) C_{\mathrm{i}}
$$




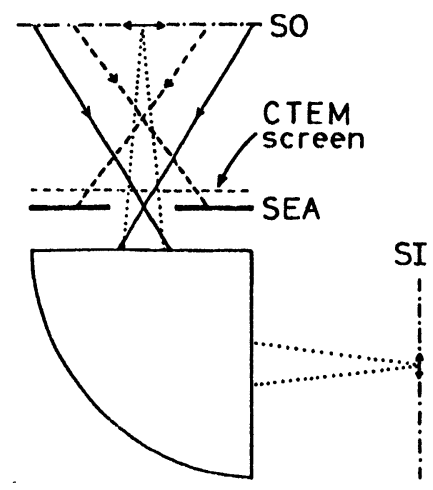

Fig. 3. - Chromatic broadening (represented by horizontal arrows) at the spectrometer object plane SO results in broadening at the spectrometer image SI. Chromatic broadening at the level of the CTEM screen may result in absorption of inelastically scattered electrons by the spectrometer entrance aperture SEA.

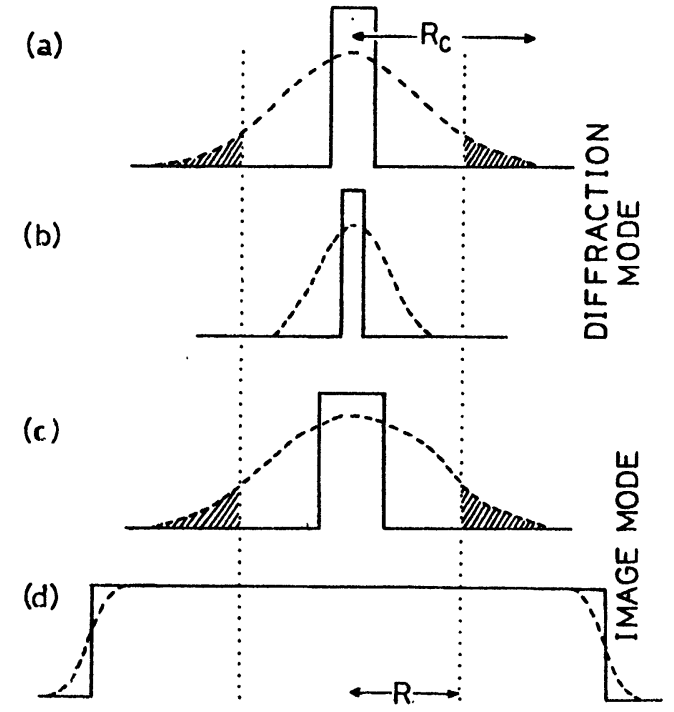

Fig. 4. - Electron-intensity profiles at the level of the spectrometer entrance aperture, without (solid lines) and with (dashed curves) chromatic aberration of postspecimen lenses. Situations (a) and (b) correspond to CTEM diffraction mode with large selected-area aperture and small selected-area aperture (or small probe), respectively. Cases (c) and (d) correspond to microscope image mode with focussed illumination (small probe) and broad illumination, respectively. The shaded areas represent loss of inelastic signal as a result of chromatic aberration.

where $d$ is the diameter of specimen contributing to the diffraction pattern, $f$ is the focal length of the objective lens, $E$ is the energy loss being analysed and $E_{0}$ is the incident-electron energy. The quantity $C_{\mathrm{i}}$, which is (within a factor of two) equal to the chromatic-aberration coefficient of the intermediate lens, has been determined experimentally to be about $30 \mathrm{~cm}$ for the Philips CM12 microscope [13]. If the specimen is broadly illuminated and $d$ is determined by a selected area (SA) aperture, $R_{\mathrm{c}}$ can exceed $R$ for large SA apertures (Fig. 4a) leading to a reduction in 
the core-loss signal [13]. But for small SA apertures or under microdiffraction conditions (where $d$ is determined by the diameter of the "nanoprobe" on the specimen), $R_{\mathrm{c}}<<R$ (Fig. 4b) and chromatic-aberration effects are negligible [14].

If an image of the specimen is present on the CTEM screen,

$$
R_{\mathrm{c}}=\beta M C_{\mathrm{o}} E / E_{0}
$$

where $C_{\mathrm{o}}$ is the chromatic-aberration coefficient of the objective lens and $M$ is the image magnification. For $E=1000 \mathrm{eV}, \beta=10 \mathrm{mrad}, E_{0}=100 \mathrm{keV}$ and $C_{\mathrm{o}}=2 \mathrm{~mm}: R_{\mathrm{c}} / M=200 \mathrm{~nm}$, so if the spectrometer entrance aperture is used to select specimen regions less than $400 \mathrm{~nm}$ in diameter, the inelastic signal may be reduced as a result of chromatic aberration (Fig. 4c). But under special ("compensating") conditions, the chromatic broadening has no effect [14]: if both the specimen and the illumination are uniform over a sufficient diameter $d$ (such that $M d>2 R+2 R_{\mathrm{c}}$ ), there is no net loss of signal within the central region defined by the aperture; see figure $4 \mathrm{~d}$.

A reduction in core-loss signal will cause an error if equation (1) is being used for absolute quantification or for measurement of cross sections [15]. More importantly: since different ionization edges correspond to differing energy loss and therefore dissimilar amounts of chromatic broadening, chromatic aberration can introduce an error in measured elemental ratios. As discussed above, such errors can be avoided by employing CTEM diffraction mode with a small selected-area aperture or a small-diameter incident beam, or by using CTEM image mode with large-diameter illumination, provided the specimen is uniform over this large area.

In the case of a highly non-uniform specimen, using a small-diameter incident probe (to define the region of analysis) avoids chromatic loss of spatial resolution, as may be required for accurate elemental ratios.

2.4 OTHER MEASUREMENT ERRORS. - Several additional factors affect the accuracy of elemental analysis by EELS. For example, drift of the specimen or of the electron probe, or radiation damage (differential mass loss) in the specimen may make the recorded data untypical of the material to be analysed. However, these effects are common to all electron-beam methods, and may actually be less severe than in EDX spectroscopy (for example) if the time and dose required for recording the EELS spectrum are lower [18].

Although the use of a parallel-recording detector greatly reduces the acquisition time needed to obtain ionization edges of adequate signal/noise ratio, these detectors suffer from certain artifacts: in particular, a channel-to-channel variation in dark current ("bias") and sensitivity (or "gain"). To avoid errors in quantification, a "bias spectrum" should be recorded and subtracted from each acquired spectrum, and the result divided by a recently-acquired "gain spectrum" [17].

\section{Procedural errors.}

The core-loss signal is weak because inner-shell excitation is a relatively improbable event. In contrast, there is a fairly high probability that a transmitted electron will be scattered elastically or inelastically by outer-shell electrons (plasmon excitation), leading to the possibility of plural (core + plasmon) scattering. Moreover, the elastic and inelastic scattering events are not independent in crystalline specimens. These physical effects, which we now consider in more detail, cause equation (1) to be only an approximation.

3.1 PluRAL inElastic SCATTERING. - Electrons which excite one or more plasmons, in addition to a core electron, shift the core-loss intensity to higher energy loss and alter the edge shape. 
Because equation (1) contains plasmon contributions to both $I_{\mathfrak{c}}$ and $I_{1}$, the value of $N$ is relatively unaffected, provided most of the plasmon-loss intensity lies within the energy window 0 to $\Delta$, i.e. if $\Delta>50 \mathrm{eV}$ for specimens which are thin enough for core-loss analysis. If this plural scattering is a problem, slightly greater accuracy may be achievable if the plural scattering is removed by deconvolution [19].

3.2 ELASTIC + INELASTIC SCATTERING. - Equation (1) assumes that elastic scattering outside the collection aperture reduces the value of $I_{c}$ and $I_{1}$ in the same proportion. This is a good approximation for amorphous specimens [1] and for large collection apertures [20], where very few electrons are scattered outside the aperture. However, the use of a large aperture reduces the mean free path for inelastic scattering [21] and, except in extremely thin specimens, increases the plural scattering contribution to the background so that edge visibility is reduced [17].

For polycrystalline or single-crystal specimens and normal-sized apertures (which exclude the Bragg-scattered electrons), $I_{c}$ contains a contribution from electrons which are first scattered elastically and then (back into the aperture) by core excitation, leading to a value of $N$ which is too large [15]. In the case of a ratio measurement, the element with the higher edge energy (for which the angular distribution of core-loss scattering is broader) will appear too high in relative concentration.

On the assumption that core-loss and elastic scattering processes are independent, equation (1) can be generalized to include the contributions of the Bragg beams:

$$
I_{\mathrm{c}}(\beta, \Delta)=N \sigma_{\mathrm{c}}(\beta, \Delta)\left[1+\sum_{\theta} b(\theta) \omega(\theta)\right]
$$

Here, $b(\theta)$ represents the intensity of a diffraction spot (or ring) at scattering angle $\theta$, relative to that of the zero-order spot, and $\omega(\theta)$ is a function which takes account of the angular distribution and the scattering geometry $[1,22]$. Use of this function has been found to improve the accuracy of elemental ratios [23], but the correction typically amounts to less than 5\% (in transmision EELS) unless the specimen is close to the Bragg orientation. It does require intensity measurements in the diffraction plane, and a simpler alternative is to try to avoid strongly diffracting conditions.

3.3 Elastic/InELASTIC COUPLiNG. - Equation (5) is based on the assumption that core-loss and elastic scattering processes are independent, so that a particular core loss has the same relative intensity and the same (Lorentzian) angular distribution around each Bragg beam. If this were generally true, the energy-filtered diffraction pattern of a single crystal would remain a spot pattern, the angular width of the spots simply increasing as the energy loss increased. In reality, energy-filtered patterns at higher energy loss more closely resemble a Kikuchi pattern, with a complex pattern of bands and lines [24, 25]. This fine structure arises from elastic scattering of high-loss (e.g. core-scattered) electrons; sometimes described as blocking [26], it is closely related (via the reciprocity principle) to electron channeling. Dynamical scattering theory, with inelastic scattering specifically included, can in principle account for these effects but requires too much detailed knowledge of the specimen (crystal structure, orientation, thickness etc.) to be useful for routine EELS analysis.

For an on-axis collection aperture, channeling and blocking effects combine to cause the coreloss intensity to vary with specimen orientation [26], equivalent to a variation of $\sigma_{\mathrm{c}}$ in equation (1). The channeling effect can be largely supressed by using an incident electron beam with a large convergence angle, as happens in the case of a small-diameter probe. Likewise, the orientation dependence due to blocking can be reduced by employing a large collection angle $\beta$, if compatible 
with edge visibility. If, in addition, the crystal is oriented so that the optic axis lies away from a major zone axis, the effects of elastic scattering will have been minimised, and the accuracy of equation (1) or equation (5), with an atomic cross section $\sigma_{\mathrm{c}}$, will be maximised.

\section{Accuracy of cross sections.}

4.1 CAlCUlATED CROSS SECTIONS. - Values of $\sigma_{\mathrm{c}}$ can be calculated from atomic-physics theory. The hydrogenic model with a Slater screening constant provides a reasonable approximation for K-shells, and is easily implemented in the form of a short computer program, designated SIGMAK [27]. For higher incident-electron energies and small values of $\beta$, relativistic kinematics should be used, as embodied in the revised program SIGMAK2 [17].

Because it somewhat overestimates core-loss intensity immediately above the ionization threshold (particularly in the lightest elements), the hydrogenic model overestimates $\sigma_{\mathrm{c}}(\beta, \Delta)$ for small values of $\Delta$. For $\Delta=100 \mathrm{eV}$, this overestimation [28] is probably less than $5 \%$ for $Z=13$ (Al), $6 \%$ to $7 \%$ for oxygen and possibly as high as $25 \%$ for $Z=3(\mathrm{Li})$. These figures are only approximate, since near-edge structure varies with the chemical and crystallographic environment of the excited atom.

For L-shells, the basic hydrogenic formalism significantly overestimates the intensity just above threshold and realistic values of $\sigma_{\mathrm{c}}(\beta, \Delta)$ require some form of empirical correction based on experimental data. In the revised computer program SIGMAL2 [17], an attempt is also made to include the contribution of white-line peaks which occur at the ionization threshold, based on electron-microscope observations [29].

More sophisticated atomic calculations use Hartree-Slater wavefunctions and are capable, in principle (i.e. with the right input parameters and with relativistic kinematics), of better accuracy. The overall shape of the edge is predicted well in many cases [30]. Because the calculations rely on atomic wavefunctions, near-edge and extended fine structures are not included. However, it has been suggested that these solid state effects change the cross section by less than $5 \%$ for $\Delta>20 \mathrm{eV}$ [31]. In some (but not all) cases, EELS measurements of $\sigma_{\mathrm{c}}$ agree more closely with Hartree-Slater cross sections than with hydrogenic-based values [32].

4.2 MEASURED CROSS SECTIONS. - Within the last few years, several groups have determined partial cross sections by EELS in a CTEM. Such measurements are valuable, not only with regard to the accuracy of the atomic models but also as a test of the magnitude of experimental errors involved in EELS microanalysis. Most measurements have involved determination of cross-section ratios (" $k$-factors") relative to a standard element, either boron [29] or oxygen [33], but some absolute values have also been reported [15].

Comparison with atomic-theory calculations sometimes shows good agreement but occasionally reveals discrepancies of as much as a factor of two [22]. The discrepancies tend to be larger for higher edge energies, and could be partly attributable to chromatic effects in postspecimen lenses, even if the measurements were performed in the CTEM diffraction mode.

In addition, values measured in different laboratories can be substantially different, as a result of dissimilar instrumentation or experimental procedures. So far, an important result of these measurements is to indicate the edges for which reliable cross sections now exist (good agreement between different experiments, or with calculations) and those for which further experimental work and/or theory is needed.

4.3 PARAMETERIZED CROSS SECTIONS. - A comparison of cross sections measured in different laboratories is complicated by the fact that different values of incident energy and collection 
angle have been employed. However, on the assuption that the aperture angle corresponds to the dipole region of scattering [17], the cross section can be expressed in terms of an integrated dipole oscillator strength $f(\Delta)$ :

$$
\sigma_{\mathrm{c}}(\beta, \Delta)=(A / T)(1 / \bar{E}) f(\Delta)\left[\ln \left(1+\beta^{2} / \bar{\theta}_{\mathrm{E}}^{2}\right)+G\left(\beta, T, \bar{\theta}_{\mathrm{E}}\right)\right]
$$

where $A=6.50 \times 10^{-14} \mathrm{~cm}^{2} \mathrm{eV}^{-2}, \bar{\theta}_{\mathrm{E}}$ is a mean characteristic scattering angle corresponding to the mean energy loss $\bar{E}=\left[E_{\mathrm{c}}\left(E_{\mathrm{c}}+\Delta\right)\right]^{1 / 2}, T$ is a relativistically-modified incident energy (expressed in $\mathrm{eV}$ ) and $G$ is a term introduced to account for relativistic retardation [22].

For typical values of $\beta(10 \mathrm{mrad}$ or less) and $\Delta(=100 \mathrm{eV})$, equation (6) can be shown to be accurate to within $7 \%$ for $\mathrm{K}$-shells of $\mathrm{Be}$ and heavier elements [22]. The relativistic term $\mathrm{G}$ changes the right-hand side of equation (6) by less than $3 \%$ at $E_{0}=100 \mathrm{keV}$, but the correction can approach $10 \%, 20 \%$ and $30 \%$ for incident energies of $200 \mathrm{keV}, 300 \mathrm{keV}$ and $400 \mathrm{keV}$ respectively (see Fig. 5) so it is a worth including in the parameterization, particularly if a computer is available to evaluate equation (6).

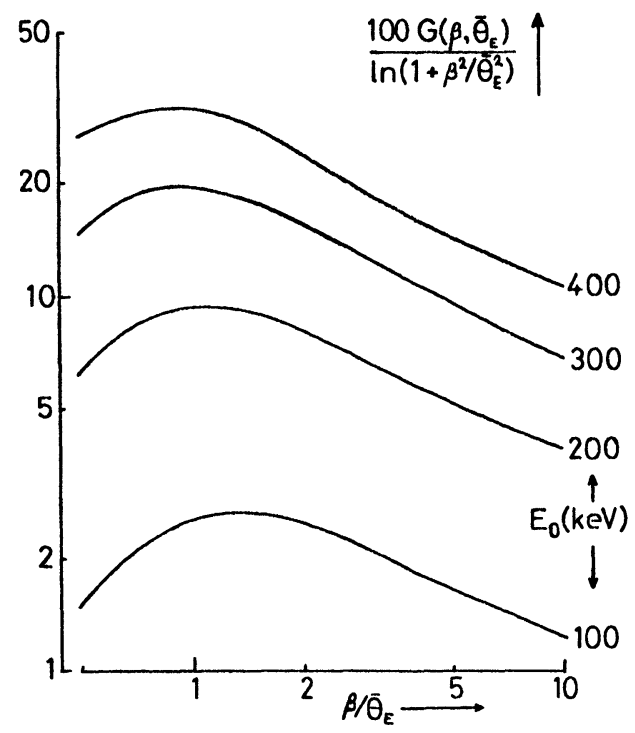

Fig. 5. - Percentage increase in $\sigma_{\mathrm{c}}(\beta, \Delta)$ due to relativistic retardation, calculated for several incident energies using equation (6).

Besides aiding the comparison of cross sections, equation (6) can provide the cross sections required for routine microanalysis, by running a simple computer code which contains stored values of $f(\Delta)$. Such a program (SIGPAR 1.0) already exists for $\Delta=100 \mathrm{eV}$ [34]. Extension to other energy windows should be straightforward, for example by storing values of $f(50 \mathrm{eV})$ and $f(200 \mathrm{eV})$, combined with interpolation.

A further advantage of this approach is that it allows data from many sources (calculations, EELS and photoabsorption experiments) to be combined to give a "best estimate" of each cross section. The stored values of $f(\Delta)$ can be updated as new data becomes available and constraints such as sum rules can be applied to check the validity of the data. 
The parameterization concept is readily extended to the case of edge fitting using equation (2). The differential cross section $\mathrm{d} \sigma_{\mathrm{c}} / \mathrm{d} E$ is given by equation (6), replacing $f(\Delta)$ by $\mathrm{d} f / \mathrm{d} E$. In order to match $\mathrm{d} \sigma_{\mathrm{c}} / \mathrm{d} E$ to measured edge profiles, an array of $\mathrm{d} f / \mathrm{d} E$ values should be stored for each edge, extending over an energy range of $100 \mathrm{eV}$ to $200 \mathrm{eV}$.

The $\mathrm{d} f / \mathrm{d} E$ spectra can be derived from calculations or from experiment. In the latter case, $\mathrm{d} f / \mathrm{d} E$ is obtained by reverse application of equation (6). If the measurements provide only the edge shape, the scaling factor can be obtained from an appropriate value of $f(100 \mathrm{eV})$.

\section{Conclusions.}

Because of the multifarious nature of the errors involved, no general figure can be placed upon the accuracy of EELS analysis; the fractional error in elemental concentration (or ratio) will depend on several detailed properties of the specimen, such as its thickness, its overall chemical composition, and the intensities, shapes and energies of the ionization edges employed for analysis, particularly in relation to other edges which are present.

However, gradual improvements in experimental technique and knowledge of cross sections are combining to increase accuracy, particularly in crystalline specimens, and to extend the technique to lower elemental concentrations.

\section{Acknowledgements.}

Recent development of EELS at Stony Brook has been supported by the National Science Foundation under grant DMR-8703516.

\section{References}

[1] EGERTON R.F., Ultramicroscopy 3 (1978) 243.

[2] EGERTON R.F., Ultramicroscopy 9 (1982) 387.

[3] BEvington P.R., Data Reduction and Error Analysis in the Physical Sciences (McGraw-Hill, New York, 1969) p. 105.

[4] PUn T., ELLIS J.R. and EDEN M., J. Microsc. 137 (1985) 93.

[5] LiU D.R. and Williams D.B., Proc. 45th Ann. Meeting EMSA (1987) 118.

[6] Colliex C., Jeanguillaume C. and Trebbia P., Microprobe Analysis of Biological Systems, T.E. Hutchinson and A.P. Somlyo Eds. (Academic Press, New York, 1981) p. 251.

[7] DE BRUIJN W.C. and SORBER W.J., Proc. XII Int. Cong. for Elec. Microsc. vol. 2 (San Francisco Press, 1990) p. 68.

[8] BENTLEY J., LEHMAN G.L. and SKLAD P.S. Analytical Electron Microscopy (San Francisco Press, 1981) p. 161.

[9] KUNDMANN M.K., in Microbeam Analysis A.D. Romig and W.F. Chambers Eds. (San Francisco Press, 1986) p. 417.

[10] STEELE J.D., TTtChMARSH J.M., ChAPMAN J.N. and PATERSON J.H., Ultramicroscopy 17 (1985) 273.

[11] LEAPMAN R.D. and SWYT C.R., Ultramicroscopy 26 (1988) 393.

[12] SHUMAN H. and SOMLYO A.P., Ultramicroscopy 21 (1987) 23.

[13] YANG Y.Y. and EGERTON R.F., Proc. XIIth International Congress for Electron Microscopy (San Francisco Press) vol. 2 (1990) p.48; research paper in preparation.

[14] TITCHMARSH J.M. and MALIS T.F., Ultramicroscopy 28 (1989) 277.

[15] Crozier P.A., Philos. Mag. B 61 (1990) 311.

[16] JoHNSON D.E., Ultramicroscopy 5 (1980) 163; 
EGERTON R.F., Ultramicroscopy 3 (1978) 39.

[17] EGERTON R.F., Electron Energy-Loss Spectroscopy in the Electron Microscope (Plenum, New York, 1986).

[18] LEAPMAN R.D., Proc. 47th Annual Meeting Electron Microsc. Soc. America (San Francisco Press, 1989) p. 400.

[19] STEPHENS A.P., Ultramicroscopy 5 (1980) 343.

[20] EGERTON R.F., Ultramicroscopy 7 (1981) 207.

[21] EGERTON R.F. and WANG Z.L., Ultramicroscopy 32 (1990) 137.

[22] EGERTON R.F., Ultramicroscopy 28 (1989) 215.

[23] WANG Z.L., J. Electron. Microsc. Technique 14 (1990) 13.

[24] Philip J.G., Whelan M.J. and EgERTON R.F., Proc. 8th Int. Cong. for Electron' Microsc. (Australian Acad. Sci., Canberra) vol. 1 (1974) p. 276.

[25] Reimer L., Proc. XIIth Int. Cong, for Electron Microsc. (San Francisco Press, 1990) p. 66.

[26] TAFTO J. and KRIVANEK O.L., Nucl. Instrum. Methods 194 (1982) 153.

[27] EGERTON R.F., Ultramicroscopy 4 (1979) 169.

[28] EgERTon R.F., Proc. 46th Ann. Meet. Elec. Microsc. Soc. Amer. (San Francisco Press, 1988) p. 532.

[29] MALIS T. and TrTCHMARSH J.M., in Electron Microscopy and Analysis 1985 (Institute of Physics, Bristol, 1985).

[30] AHN C.C. and REZ P., Ultramicroscopy 17 (1985) 105.

[31] WENG X. and REZ P., Ultramicroscopy 25 (1988) 345.

[32] TREBBIA P. and MANOUBI T., Ultramicroscopy 28 (1989) 266.

[33] HOFER F. and Golub P., Micron. Microsc. Acta 19 (1988) 73;

HOFER F., GOLUB P. and BRUNEGGER A., Ultramicroscopy 25 (1988) 81;

HOFER F., J. Microsc. 156 (1989) 279.

[34] A listing of this program is available from the author. 